Bouwman, A. F. (Hrsg.), 1990: Soils and the greenhouse effect. International conference held Aug. 14-18, 1989, Wageningen, Netherlands. 575 S., Wiley, Chichester

Bouwman, A.F., 1996: Direct emission of nitrous oxide from agricultural soils. Nutrient Cycling in Agroecosystems 46: 53-70

BRUHL, C. (1993): The impact of the future scenarios for methane and other chemically active gases on the GWP of methane. Chemosphere 26: 731-738

DIN (Deutsches Institut für Normung; Hrsg.), 1996: DIN EN ISO 14040: Produkt-Ökobilanz, Prinzipien und allgemeine Anforderungen, Deutsche Fassung prEN ISO 14040, Normenausschuß Grundlagen des Umweltschutzes (NAGUS) im DIN Deutsches Institut für Normung e. V., Berlin

Hejuncs, R. (Hrsg.), 1992: Environmental life cycle assessment of products. Guide (Part 1), Centrum voor Milieukunde, Leiden 1992

IPCC, 1996: Intergovernmental Panel of Climate Change: Climate Change 1995 - The Science of Climate Change. Cambridge University Press, Cambridge/UK

IPCC, 1997: Intergovernmental Panel of Climate Change: Revised 1996 IPCC Guidelines for National Greenhouse Gas Inventories: Workbook. Module 4: Agriculture. http://www.iea.org/ipcc/ general/invs $5 \mathrm{c} . \mathrm{htm}$

ISERMANN, K., 1990: Ammoniakemission der Landwirtschaft als Bestandteil ihrer Stoffbilanz und Lösungsansätze zur Minderung. In: Kuratorium für Technik und Bauwesen in der Landwirtschaft (Hrsg.): Ammoniak in der Umwelt - Kreisläufe, Wirkungen, Minderung. VDI/KTBL-Symposium 10.-12. Oktober 1990, Landwirtschaftsverlag, Münster-Hiltrup

KalTsCHMITT, M., 1997: Biomasse: Was kann sie zur Treibhausgasminderung beitragen? Arbeitsgruppe Luftreinhaltung der Universität Stuttgart, Jahresbericht 1996/1997: Luftreinhaltung in Baden-Württemberg: Das Treibhausgas $\mathrm{CO}_{2}$ - Wirkung und Minderung, 122-138

KaltSCHMIT, M., A. WIESE (Hrsg.), 1997: Erneuerbare Energien: Systemtechnik, Wirtschaftlichkeit, Umweltaspekte. Springer, Berlin/Heidelberg, 2. Auflage
Kaltschmitt, M., G. A. Reinhardt (Hrsg.), 1997: Nachwachsende Energieträger. Grundlagen, Verfahren, Ökologische Bilanzierung. Vieweg, Braunschweig/Wiesbaden

Lammel, G., H. Grassl (1995): Greenhouse effect of $\mathrm{NO}_{x}$. Environ.Sci. \& Pollut. Res. 2: 40-45

NusSBaUmer, T., 1989: Schadstoffbildung bei der Verbrennung von Holz. Forschungsbericht Nr. 6, Laboratorium für Energiesysteme, ETH Zürich, Juris, Zürich

Projektgemeinschaft Bioenergieträger: Institut für Energiewirtschaft und Rationelle Energienanwendung (IER). Universität Stuttgart; IFEU - Institut für Energie- und Umweltforschung Heidelberg $\mathrm{GmbH}$, Institut für Umweltstudien (IUS), Heidelberg; Kuratorium für Technik und Bauwesen in der Landwirtschaft (KTBL), Darmstadt

SETAC (Society of Environmental Toxicology and Chemistry, Hrsg.), 1993: Guidelines for Life Cycle Assessment: A Code of Practice. Agreement of a working group of SETAC, SETAC, Brüssel

SkIBA, U., K. A. SmITH, D. Fowler, 1993: Nitrification and denitrification as sources of nitric oxide and nitrous oxide in a sandy loam soil. Soil Biology and Biochemistry 25: 1527-1536

Statistisches Bundesamt, 1996: Statistisches Jahrbuch der Bundesrepublik Deutschland 1996, Metzler-Poeschel, Stuttgart

Stohl, A., E. Williams, G. Wotawa, H. Kromp-Kolb, 1996: A European inventroy of soil nitric oxide emissions and the effect of these emissions on the photochemical formation of ozone. Atmospheric Environment 30: 3741-3755

UBA (Umweltbundesamt), 1995: UBA-Texte 23/95, Methodik der produktbezogenen Ökobilanzen. Wirkungsbilanz und Bewertung, UBA, Berlin

Williams, E. J., G. L. Hutchinson, F. C. Fehsenfeld, 1992: NO and $\mathrm{N}_{2} \mathrm{O}$ emissions from soil. Global Biogeochemical Cycles 6 : 351-388

Yamulki, S., K. W. T. Goulding, C. P. Webster, R. M. Harrison, 1995: Studies on $\mathrm{NO}$ and $\mathrm{N}_{2} \mathrm{O}$ fluxes from a wheat field. Atmospheric Environment 29: 1627-1635

\title{
Erratum
}

UWSF - Z. Umweltchem. Ökotox. 10 (5) 271-275 (1998)

\section{Bakterielle Biotestverfahren zur Bestimmung der Toxizität von Abfällen}

\author{
U. Stotmann, J. Roll, C. Gendig, S. Broja, H. Czycholl
}

In Tabelle 4, Spalte Abfall C ist der Wert in Zeile EPA 1320 EC20 [m//l] fälschlicherweise mit " 7500" " angegeben. Die korrekte Angabe lautet " $>500^{a}$. 